\title{
ER-to-Golgi transport and SEC23-dependent COPII vesicles regulate $T$ cell alloimmunity
}

\author{
Stephanie Kim, ${ }^{1,2}$ Rami Khoriaty, ${ }^{1,3}$ Lu Li, ${ }^{1}$ Madison McClune, ${ }^{1}$ Theodosia A. Kalfa, ${ }^{4,5}$ Julia Wu, ${ }^{1,2}$ Daniel Peltier, ${ }^{6}$ Hideaki Fujiwara, \\ Yaping Sun, ${ }^{1}$ Katherine Oravecz-Wilson, ${ }^{1}$ Richard A. King, ${ }^{1}$ David Ginsburg, ${ }^{1,6,7,8,9,10}$ and Pavan Reddy ${ }^{1}$ \\ 'Department of Internal Medicine, Division of Hematology and Oncology, ${ }^{2}$ Medical Scientist Training Program, and ${ }^{3}$ Department of Cell and Developmental Biology, University of Michigan, Ann Arbor, \\ Michigan, USA. ${ }^{4}$ Cancer and Blood Diseases Institute, Cincinnati Children's Hospital Medical Center, Cincinnati, Ohio, USA. ${ }^{5}$ Department of Pediatrics, University of Cincinnati College of Medicine, Cincinnati, \\ Ohio, USA. ${ }^{6}$ Department of Pediatrics, Division of Hematology and Oncology, ${ }^{7}$ Department of Human Genetics, ${ }^{8}$ Life Sciences Institute, ${ }^{9}$ Howard Hughes Medical Institute, and ${ }^{10} \mathrm{Department}$ of Internal \\ Medicine, Division of Genetic Medicine, University of Michigan, Ann Arbor, Michigan, USA.
}

T cell-mediated responses are dependent on their secretion of key effector molecules. However, the critical molecular determinants of the secretion of these proteins are largely undefined. Here, we demonstrate that $T$ cell activation increases trafficking via the ER-to-Golgi pathway. To study the functional role of this pathway, we generated mice with a T cell-specific deletion in SEC23B, a core subunit of coat protein complex II (COPII). We found that SEC23B critically regulated the T cell secretome following activation. SEC23B-deficient $\mathrm{T}$ cells exhibited a proliferative defect and reduced effector functions in vitro, as well as in experimental models of allogeneic and xenogeneic hematopoietic cell transplantation in vivo. However, T cells derived from 3 patients with congenital dyserythropoietic anemia II (CDAII), which results from Sec23b mutation, did not exhibit a similar phenotype. Mechanistic studies demonstrated that unlike murine KO T cells, T cells from patients with CDAll harbor increased levels of the closely related paralog, SEC23A. In vivo rescue of murine KO by expression of Sec23a from the Sec23b genomic locus restored T cell functions. Together, our data demonstrate a critical role for the COPII pathway, with evidence for functional overlap in vivo between SEC23 paralogs in the regulation of T cell immunity in both mice and humans.

\section{Introduction}

Coat protein complex II (COPII) drives the formation of ERderived vesicles that transport newly synthesized proteins to the Golgi compartment. Among their critical roles, COPII-coated vesicles enable the extracellular transport of proteins and their localization to other intracellular compartments (1-4). Structurally, COPII is comprised of an inner and outer coat whose core components are conserved in all eukaryotes (5). SEC23 is a core subunit of the COPII inner layer $(6,7)$. It is required for the selection and incorporation of cargo molecules through its interaction with inner coat protein SEC24 (8-11) and is essential for COPIIdependent anterograde protein trafficking $(6,12,13)$. While the fundamental COPII pathway is conserved in all eukaryotes, the presence of multiple COPII subunit paralogs in mammals enables increased complexity and regulation of protein secretion $(5,14)$. The mammalian genome encodes for 2 SEC23 paralogs, SEC23A and SEC23B. These paralogs share $85 \%$ sequence identity at the amino acid level (15). Yet, mutations in $S e c 23 a$ and $S e c 23 b$ paralogs result in 2 different disorders in humans, cranio-lenticulo-sutural dysplasia (CLSD) $(16,17)$ and congenital dyserythropoietic anemia type II (CDAII) (18), respectively. The definitive roles of these paralogs in vivo, and in immunity, however, remain unknown.

Conflict of interest: The authors have declared that no conflict of interest exists. Copyright: () 2021, American Society for Clinical Investigation.

Submitted: January 21, 2020; Accepted: November 12, 2020; Published: January 19, 2021. Reference information: J Clin Invest. 2021;131(2):e136574.

https://doi.org/10.1172/JCl136574.
Naive T cells undergo a program of increased protein synthesis following their activation, which includes the production of secretory proteins that facilitate $\mathrm{T}$ cell growth and effector functions. The intracellular pathways and molecular determinants that regulate them remain poorly characterized $(19,20)$. Specifically, the pathways of protein transport prior to their access to the Golgi compartment and whether they regulate $\mathrm{T}$ cell functions are not known.

Herein we characterize ER-to-Golgi transport mechanisms that are critical for $\mathrm{T}$ cell functions in vitro and in vivo. We demonstrate for the first time to our knowledge that unlike other hematopoietic cells, naive T cells from mice and humans express both SEC23 paralogs and harbor greater baseline expression of SEC23B than SEC23A. Conditional hematopoietic- and T cell-specific KO models of both paralogs of SEC23 demonstrated a key role for SEC23B in WT T cells, but that neither paralog alone is critical for $\mathrm{T}$ cell development. The loss of $\operatorname{Sec} 23 b$ led to reduced secretion of select effector cytokines, altered functions of murine and human $\mathrm{T}$ cells in vitro, and reduced pathogenicity in murine in vivo models of allogeneic hematopoietic stem cell transplantation (allo-HCT) and xenogeneic graft-versus-host disease (GVHD). However, introduction of the Sec23a coding sequence in the Sec23b genomic locus restored $\mathrm{T}$ cell functions in murine $\mathrm{T}$ cells, as was also observed in human $\mathrm{T}$ cells when SEC23A was increased. Our findings demonstrate that SEC23 paralogs have overlapping functions in $\mathrm{T}$ cells in vivo when expressed at similar levels, and that the COPII pathway is critical for $\mathrm{T}$ cell immunity. 
A
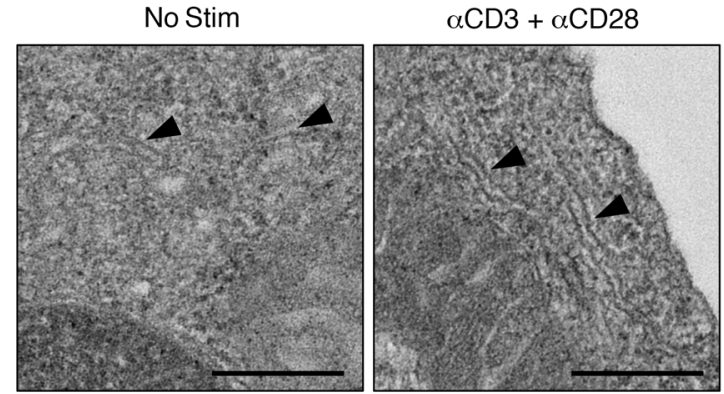

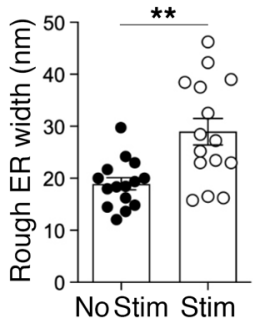

C
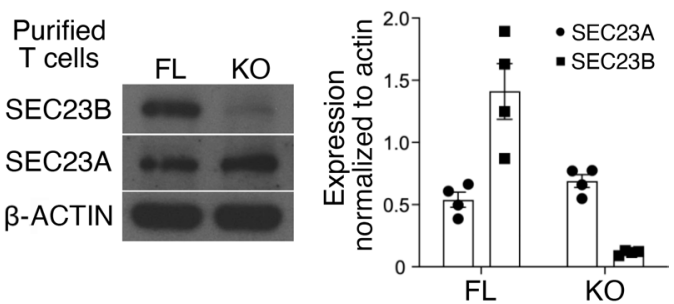

Eif2a $\overline{\text { Atf6a }} \overline{\text { Grp94 }} \overline{\mathrm{BiP}}$

D

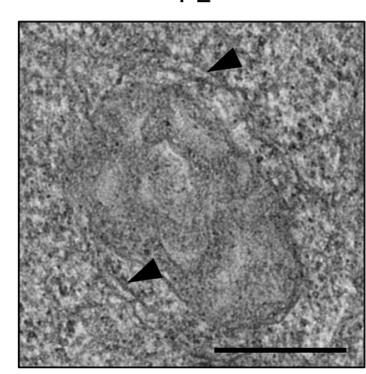

FL
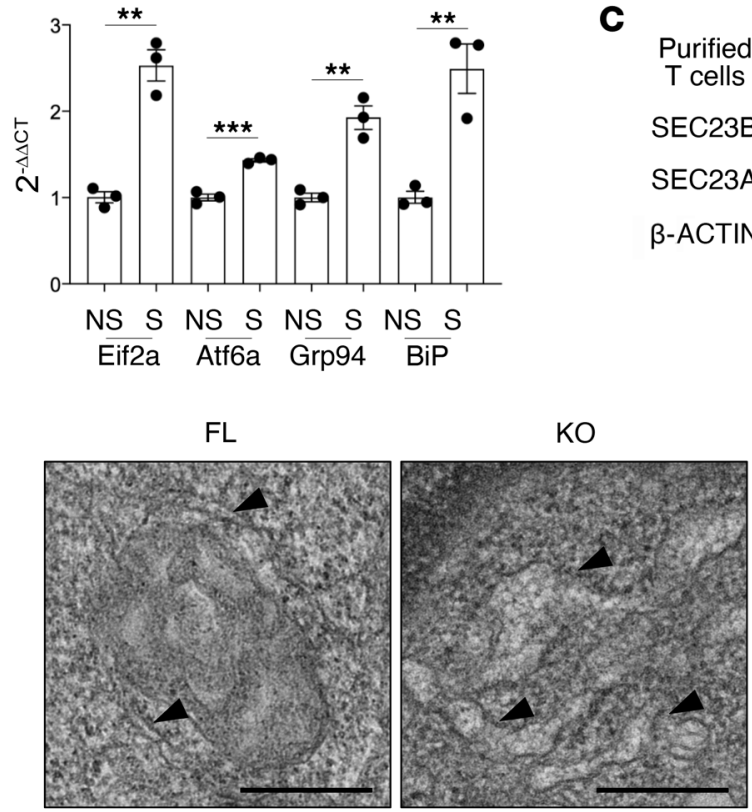

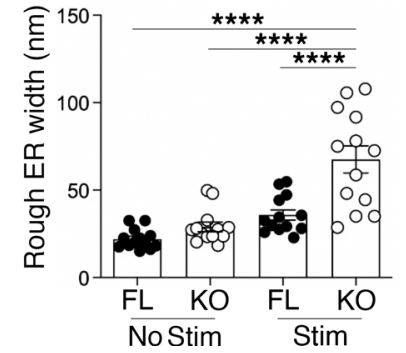

Figure 1. COPII-dependent ER-to-Golgi pathway is engaged upon T cell activation. (A) Transmission electron micrographs of WT murine T cells that received no stimulation or stimulation with $\alpha \mathrm{CD} 3$ and $\alpha \mathrm{CD} 28$ for 3 days. Measurements of rough ER widths were taken from 3 different ERs from 5 cells in each group $(n=15)$. Scale bars: $100 \mathrm{~nm}$. (B) Real-time quantitative PCR analysis of ER stress-associated genes in WT murine T cells that received no stimulation or 24 hours of stimulation with $\alpha \mathrm{CD} 3$ and $\alpha \mathrm{CD} 28(n=3)$. (C) Western blots showing protein levels of SEC23B, SEC23A, and $\beta$-actin in Sec23b ${ }^{f / /}$ Cd4 ${ }^{\mathrm{Cre}}(\mathrm{KO}) \mathrm{T}$ cells relative to Sec23b $b^{f /-}(\mathrm{FL})$ controls. Densitometric analysis on the right plot indicates normalized levels of SEC23A and SEC23B relative to

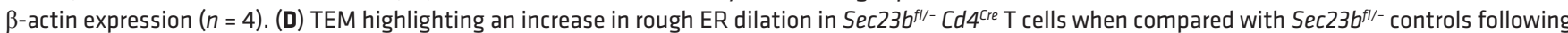
activation with $\alpha \mathrm{CD} 3$ and $\alpha \mathrm{CD} 28$ for 3 days $(n=13)$. Scale bars: $100 \mathrm{~nm}$. (A and B) Data represent mean $\pm \operatorname{SEM}$, with ${ }^{* *} P<0.01$ and ${ }^{* * *} P<0.001(2$-tailed unpaired Student's $t$ test). (D) Statistical significance was determined by 1-way ANOVA and post hoc Tukey's test; ${ }^{* * *} P<0.0001$.

\section{Results}

The COPII-dependent ER-to-Golgi pathway is engaged upon T cell activation. To determine whether the ER-to-Golgi pathway is utilized by $\mathrm{T}$ cells following activation, we first examined naive WT $\mathrm{T}$ cells by transmission electron microscopy (TEM) before and after activation with $\alpha \mathrm{CD} 3$ and $\alpha \mathrm{CD} 28$ antibodies, and determined rough ER morphology in a blinded manner. Unstimulated $\mathrm{T}$ cells exhibited narrow cisternae of the rough ER typical of resting cells. Following activation, the rough ER demonstrated a significant dilation of the cisternae (Figure 1A). This change was associated with increased induction of the ER stress-associated genes Eif2a, Atf6a, Grp94, and Bip, demonstrating engagement of the ER-to-Golgi pathway (Figure 1B). We next hypothesized that increased ER trafficking of proteins following $\mathrm{T}$ cell activation may lead to the transport of these proteins to the Golgi compartment via COPII vesicles.
To test this, we examined the consequence of abrogating COPII-dependent protein transport following naive $\mathrm{T}$ cell activation. To this end, we generated $\operatorname{Sec} 23 b^{f /-} \mathrm{Cd} 4^{\mathrm{Cre}}$ mice, which harbor a $\mathrm{T}$ cell-specific deficiency in SEC23B (Figure 1C). To rule out Cre-specific artifacts, we also generated $\operatorname{Sec} 23 b^{f /-}$ Vav $1^{\mathrm{Cre}}$ mice, which carry a hematopoietic cell-specific SEC23B deficiency, to validate the results (Supplemental Figure 1); supplemental material available online with this article; https://doi.org/10.1172/ JCI136574DS1). We reasoned that in the absence of SEC23B, T cells would demonstrate a greater increase in distension of the rough ER cisternae secondary to accumulation of COPII cargo proteins. Compared with $\operatorname{Sec} 23 b^{f /-}$ controls, $\operatorname{Sec} 23 b^{f /-} C d 4^{\text {Cre }} \mathrm{T}$ cells displayed significantly distended rough ER as measured by TEM, both when unstimulated and following stimulation with $\alpha \mathrm{CD} 3$ and $\alpha \mathrm{CD} 28$ antibodies (Figure 1D). Similar results were observed in $\operatorname{Sec} 23 b^{f /-} \operatorname{Vav}^{\mathrm{Cre}} \mathrm{T}$ cells (data not shown). These data demonstrate 
A
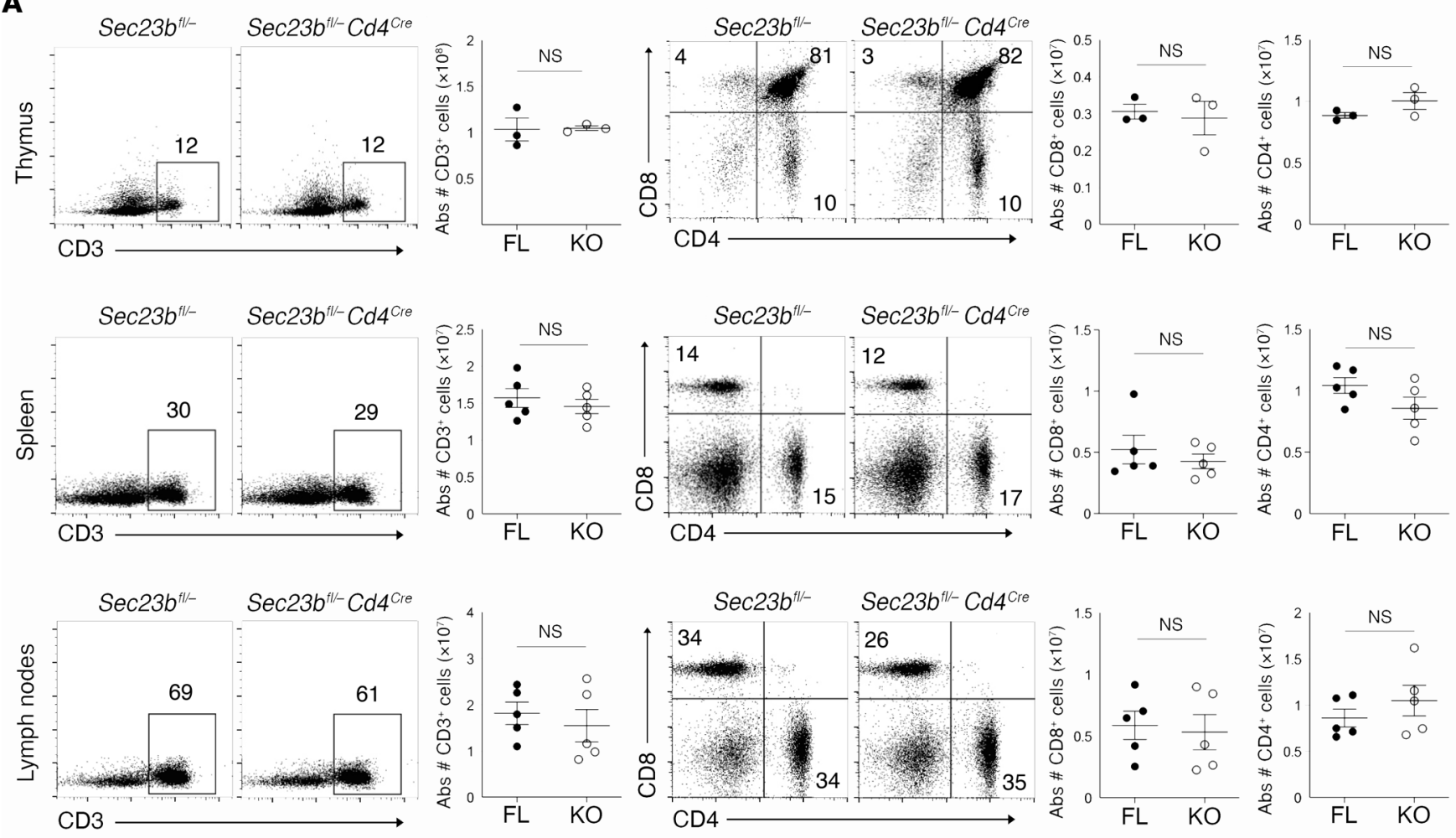

B

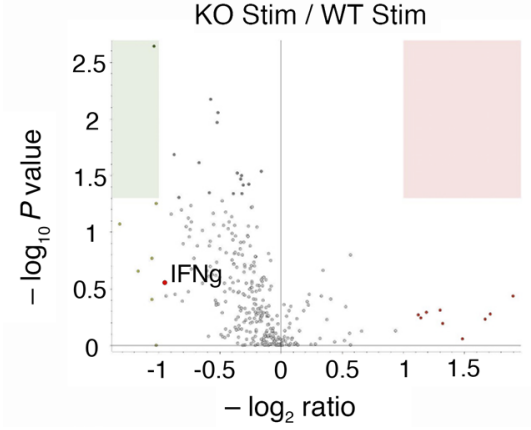

C

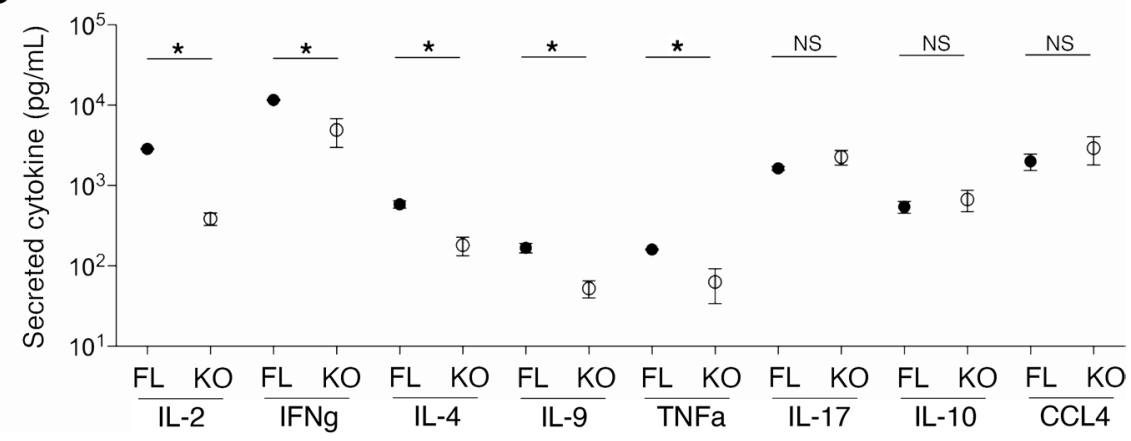

Figure 2. SEC23B deficiency does not affect mature T cell development but regulates its secretome. (A) Percentages and absolute numbers of Sec $23 b^{f l /-}$ $(\mathrm{FL})$ and $\operatorname{Sec} 23 b^{f / /} C d 4^{\mathrm{Cre}}(\mathrm{KO}) \mathrm{CD} 3^{+} \mathrm{T}$ cells in whole thymus $(n=3)$, spleen $(n=5)$, and lymph node $(n=5)$ homogenate samples (left), and those of CD4 ${ }^{+}$and $\mathrm{CD}^{+} \mathrm{T}$ cell subsets gated on CD3+ cells (right). (B) Volcano plot representing 3 biological replicates of SEC23B-deficient T cell secretomes after 24 hours of stimulation compared with WT. $\log _{2}$ ratios were calculated based on $P$ values. (C) Luminex panel showing mean cytokine levels derived from Sec23b $b^{f /-}$ (FL) and $\operatorname{Sec} 23 b^{f l /}-C d 4^{\text {Cre }}(\mathrm{KO})$ T cell supernatants following 3 days of stimulation with $\alpha \mathrm{CD} 3$ and $\alpha \mathrm{CD} 28$. (A and $\left.\mathbf{C}\right)$ Data represent mean $\pm \mathrm{SEM}$, with ${ }^{*} P<0.05$ (2-tailed unpaired Student's $t$ test).

that $\mathrm{T}$ cells depend on adequate levels of SEC23B-dependent COPII formation for synthesized proteins to exit the ER.

SEC23B deficiency does not affect mature $T$ cell development but regulates its secretome. Because SEC23B-dependent COPII formation appeared to be essential for the efficient transport of proteins from the ER in activated $\mathrm{T}$ cells, we next determined whether SEC23B was critical for T cell development. We examined both $\operatorname{Sec} 23 b^{f /-} C d 4^{\mathrm{Cre}}$ and $\operatorname{Sec} 23 b^{\mathrm{fl} / \mathrm{Vav}} 1^{\mathrm{Cre}}$ murine models and found that absolute numbers and ratios of thymic subsets (double negative, double positive, single positive) and mature $\mathrm{T}$ cell subsets from spleen and lymph nodes from both models were similar when compared with age matched WT animals, suggest- ing that deficiency of Sec $23 b$ did not affect $\mathrm{T}$ cell development (Figure 2A, and Supplemental Figure 2). We next tested whether disrupting SEC23B-mediated COPII formation would impact the profile of secreted proteins following $\mathrm{T}$ cell activation. To enable high-throughput identification of secreted proteins, we analyzed the $\mathrm{T}$ cell secretome with LC-MS/MS. We stimulated WT and $\operatorname{Sec} 23 b^{f /-} C d 4^{C r e}$ naive T cells with $\alpha \mathrm{CD} 3$ and $\alpha \mathrm{CD} 28$, and enriched the protein fraction in the supernatant for mass spectrometric analysis. Activated $\operatorname{Sec} 23 b^{f /-} C d 4^{\mathrm{Cre}} \mathrm{T}$ cell supernatants exhibited a gross reduction in the amount of secreted proteins when compared with that of WT T cells. Among the reduced secreted proteins were those involved in the inflammatory response such as 
A

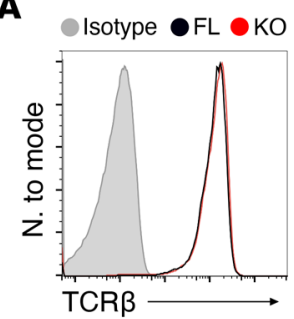

B

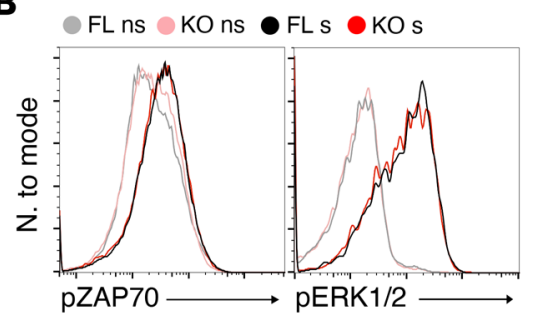

C

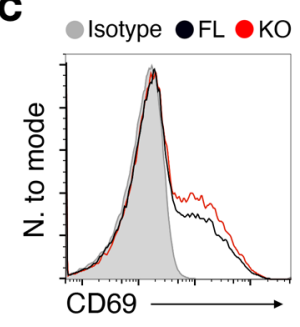

D

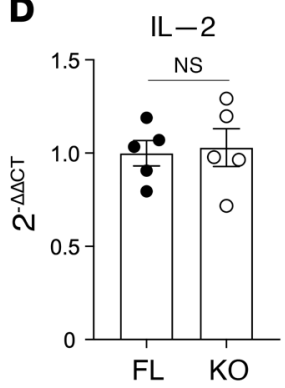

E
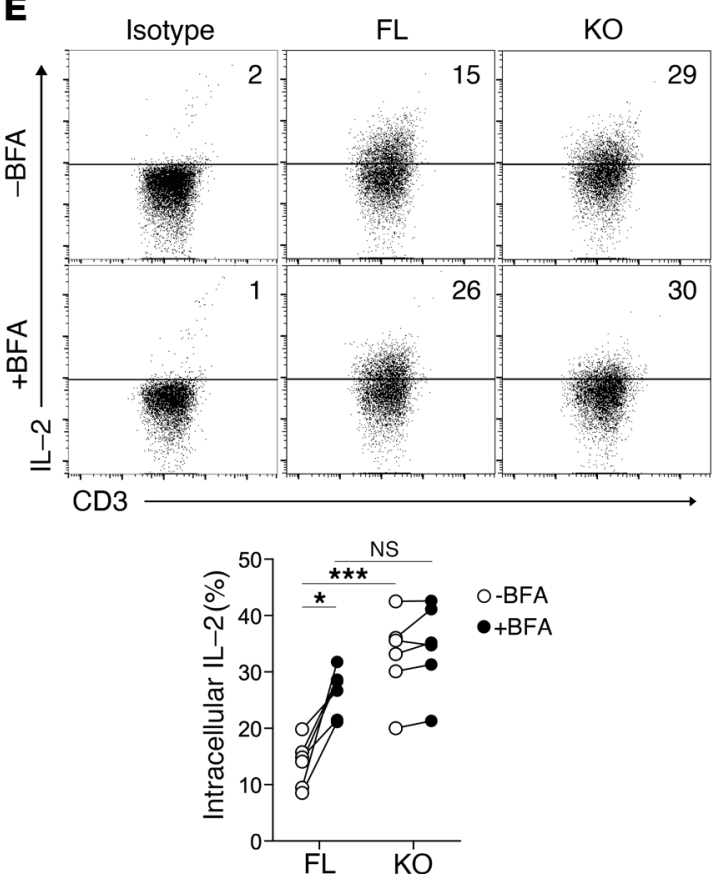

$\mathbf{F}$

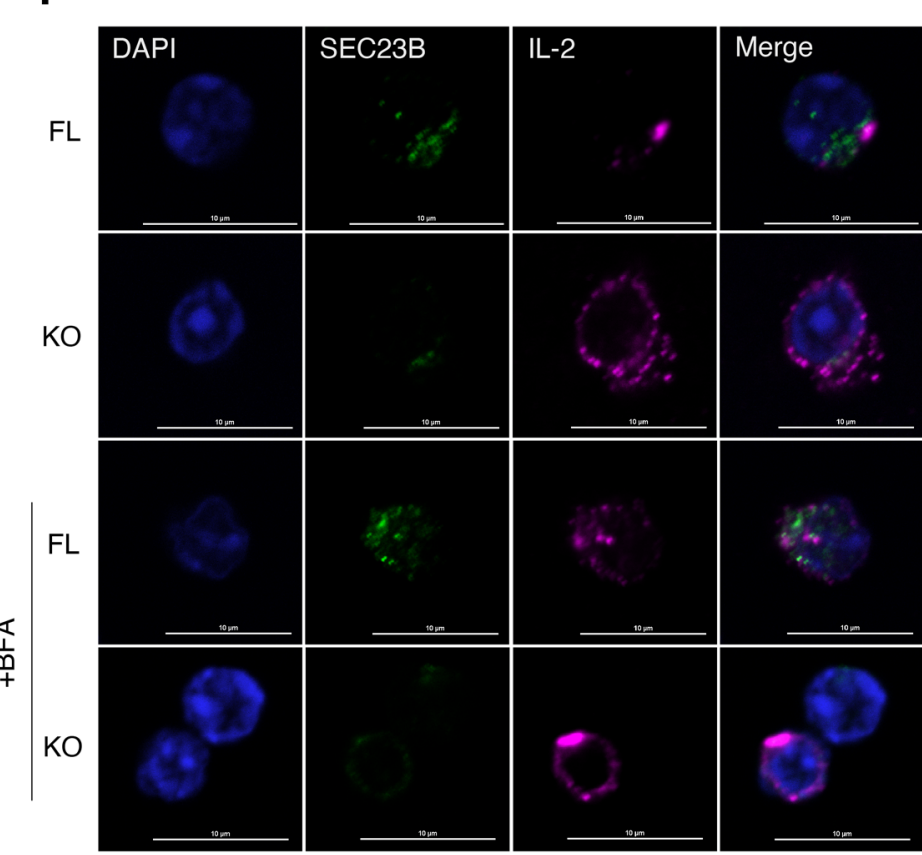

Figure 3. Deficiency of SEC23B-dependent COPII leads to accumulation of secreted proteins. (A) Histograms based on flow cytometry of surface TCR- $\beta$ on Sec $23 b^{f /-}$ and $\operatorname{Sec} 23 b^{f /-}$ Cd $4^{\text {cre }}$ T cells relative to isotype levels present on naive unstimulated WT T cells. (B) Flow cytometry of phosphorylated ZAP70 and ERK1/2 molecules in Sec23b $b^{f /-}$ and Sec23b $b^{f /-}$ Cd4 $4^{\text {Cre }}$ T cells that received no stimulation or stimulation with $\alpha$ CD3 and $\alpha$ CD28 for 30 minutes. (C) Flow cytometry of surface CD69 present on Sec23b $b^{f /-}$ and Sec23bfl/- Cd4 $4^{\text {Cre }}$ T cells stimulated with $\alpha$ CD3 and $\alpha$ CD28 for 6 h compared with isotype levels present on naive unstimulated WT T cells. Flow cytometric data are representative of 3 replicate experiments. (D) qRT-PCR analysis of IL-2 in SEC23B-deficient T cells compared with WT following stimulation with $\alpha$ CD3 and $\alpha$ CD28 for 4 days ( $n=5 /$ group). (E) Flow cytometry measuring intracellular IL-2 levels in T cells after stimulation by $\alpha \mathrm{CD} 3$ and $\alpha \mathrm{CD} 28$ for 3 days followed by 5 hours of stimulation with PMA and ionomycin in the presence or absence of BFA ( $n=6 /$ group). (F) Immunofluorescence confocal micrographs of SEC23B (green) and IL-2 (pink) in WT or SEC23B-deficient T cells after 3 days of $\alpha \mathrm{CD} 3$ and $\alpha \mathrm{CD} 28$ stimulation, and 5 hours of PMA and ionomycin stimulation with or without BFA. (D) Data represent mean \pm SEM, and significance was determined by 2 -tailed unpaired Student's $t$ test. (E) Statistical significance was determined by 1-way ANOVA and post hoc Tukey's test; ${ }^{*} P<0.05,{ }^{* *} P<0.001$. Scale bars: $10 \mu \mathrm{M}$.

IFN- $\gamma$ (Figure 2B). Next, we validated this protein secretion pattern by focusing on cytokines utilizing a multiplex assay, and confirmed decreased levels of IFN- $\gamma$ and other cytokines such as IL-2 and TNF- $\alpha$ in SEC23B-deficient T cell supernatants. Of note, the secretion of few other cytokines by SEC23B-deficient $\mathrm{T}$ cells was similar to that by WT T cells, indicating that reduced secretion of cytokines by COPII-deficient $\mathrm{T}$ cells was not a global phenomenon (Figure 2C).

Deficiency of SEC23B-dependent COPII leads to accumulation of secreted proteins. Reduction in secreted proteins could be secondary to differences in activation of $\mathrm{T}$ cells in the absence of SEC23B. Therefore, we next analyzed whether the reduction in the secretome was secondary to defects in $\mathrm{T}$ cell activation. When compared with WT T cells, Sec $23 b^{f /-} C d 4^{\text {Cre }} \mathrm{T}$ cells expressed sim- ilar levels of surface TCR- $\beta$ (Figure 3A) and phosphorylated TCR signaling components such as ZAP-70 and ERK1/2 (Figure 3B) following activation. Furthermore, $\operatorname{Sec} 23 b^{f /-} C d 4^{\text {Cre }} \mathrm{T}$ cells expressed similar levels of the activation marker CD69 after stimulation (Figure 3C). These data suggest that abrogating SEC23B-dependent COPII formation does not affect TCR activation.

We next determined whether the reduction of secreted proteins in the supernatants is secondary to defects in protein synthesis, with a focus on IL-2. SEC23B-deficient T cells expressed normal levels of IL-2 at the mRNA level post-stimulation with $\alpha \mathrm{CD} 3$ and $\alpha \mathrm{CD} 28$ (Figure $3 \mathrm{D}$ ), suggesting that $\mathrm{T}$ cell activation was intact, and that the SEC23B-deficient $\mathrm{T}$ cells were able to produce but unable to secrete IL-2. To confirm this, we stimulated cytokine production in T cells with PMA and ionomycin in the presence or 
absence of Brefeldin A (BFA), an inhibitor of anterograde transport between the ER and Golgi apparatus (21). In the presence of BFA, both WT and SEC23B-deficient T cells demonstrated similar levels of intracellular IL-2 following activation (Figure 3E), suggesting that the absence of SEC23B does not impact IL-2 protein synthesis. By contrast, WT T cells contained less intracellular IL-2 in the absence of BFA than in the presence of BFA, suggesting that some intracellular IL-2 was secreted by WT T cells in a COPIIdependent manner. However, in SEC23B-deficient $\mathrm{T}$ cells, the intracellular levels of IL-2 remained high despite the absence of BFA in culture (Figure 3E).

To further confirm SEC23B expression levels and to localize IL-2 intracellularly, we examined T cells by immunofluorescence confocal microscopy. T cells lacking SEC23B displayed greater accumulation of intracellular IL-2 when compared with WT cells, and importantly, in WT cells, IL-2 colocalized with SEC23B (Figure $3 \mathrm{~F}$ ). Together, these data demonstrate that SEC23B-dependent COPII-mediated ER-to-Golgi transport is critical for the release of T cell-derived proteins such as IL-2.

SEC23B-dependent COPII regulates naive $T$ cell functions in vitro. Given the importance of cytokine secretion for T cell-mediated functions, we next investigated the functional consequences of abrogating the COPII pathway in T cells. Because of the differential effects observed on cytokine release, we first determined the impact of SEC23B deficiency on the ability of naive $\mathrm{CD} 4^{+} \mathrm{T}$ cells to differentiate into helper $\mathrm{T}$ subsets. When cultured in polarizing media, naive $S e c 23 b^{f /-} \mathrm{Cd} 4^{\mathrm{Cre}} \mathrm{CD} 4^{+} \mathrm{T}$ cells exhibited a normal capacity to differentiate into Th1, Th2, and Th17 cells as measured by lineage-specifying transcription factors T-bet, GATA3, and ROR $\gamma \mathrm{t}$, respectively (Figure 4A).

We next analyzed the impact of SEC23B deficiency on T cell proliferation in vitro. $\mathrm{T}$ cells were stimulated with $\alpha \mathrm{CD} 3$ and $\alpha \mathrm{CD} 28$ for 3 days following staining with CFSE. SEC23Bdeficient $\mathrm{T}$ cells exhibited reduced proliferative capacity when compared with WT T cells as measured by CFSE dilutions (Figure 4B). Proportions of Annexin V and 7-AAD were similar in SEC23B-deficient and WT T cells (Figure 4C), suggesting that this observation was primarily due to defects in proliferation and not secondary to increased cell death. We next examined whether the proliferation defect was secondary to decreased COPII-dependent secretion of proteins such as IL-2, a critical $\mathrm{T}$ cell growth factor. The addition of exogenous recombinant murine IL-2 partially corrected the proliferation defect in vitro, suggesting that the altered secretome, specifically the release of IL-2, contributed only in part to the reduction in proliferation (Figure 4B). These data demonstrate that SEC23B regulates in vitro $\mathrm{T}$ cell proliferation, but not differentiation.

SEC23B-dependent COPII regulates allogeneic $T$ cell functions. To determine the relevance of SEC23B-dependent effects on $\mathrm{T}$ cells in vivo, we utilized a well-defined model of $\mathrm{T}$ cell stimulation, experimental allo-HCT. We hypothesized that SEC23B deficiency in donor $\mathrm{T}$ cells would result in reduced proliferation, and confer reduced pathogenicity in the clinically relevant major histocompatibility-mismatched [C57BL/6J $\rightarrow$ BALB/c] model of allo-HCT. We first tracked $\mathrm{T}$ cell proliferation in vivo by transferring CFSE-stained WT or SEC23B-deficient T cells into allogeneic $\mathrm{BALB} / \mathrm{c}$ mice that received total body irradiation (TBI) on day -1 , and analyzing recipient spleens 3 days after transplant. Allogeneic SEC23B-deficient $\mathrm{T}$ cells proliferated significantly less than WT T cells, and significantly lower numbers were recovered in the spleen (Figure 4D), demonstrating an in vivo proliferative defect similar to in vitro responses. When we tested the allo-stimulated $\mathrm{T}$ cells in a ${ }^{51}$ Chromium release assay, we also found that SEC23B-deficient $\mathrm{T}$ cells have reduced cytotoxicity against allogeneic target cells compared with WT T cells (Figure 4E). We therefore next analyzed the pathogenic capacity of SEC23B-deficient $\mathrm{T}$ cells to induce GVHD.

Donor $\mathrm{T}$ cells are essential for driving GVHD in recipient mice, and the network of cytokines secreted by $\mathrm{T}$ cells are both important in the initiation and maintenance of GVHD (22). To test whether the proliferative defect of SEC23B-deficient $\mathrm{T}$ cells impacted GVHD pathogenesis in vivo, we assessed their ability to drive murine $\operatorname{GVHD}(23,24)$. Specifically, C57BL/6J WT or SEC23B-deficient T cells were combined with C57BL/6J WT bone marrow and transferred into allogeneic BALB/c and syngeneic $\mathrm{C} 57 \mathrm{BL} / 6 \mathrm{~J}$ recipient mice. All recipients of syngeneic T cells survived, while all recipients of allogeneic WT T cells died by 9 weeks after transplant (Figure 4F). However, recipients of allogeneic SEC23B KO T cells demonstrated a dramatic improvement in survival with only $6 \%$ mortality. Clinical GVHD scores in these recipients were significantly lower than those of recipients of WT T cells during the posttransplant period (Figure $4 \mathrm{~F}$ ). To rule out strain-dependent artifacts, we next corroborated these data in a second MHC matched and minor histocompatibility-mismatched model of allo-HCT using C3H.SW recipients [C57BL/6J $\rightarrow$ C3H.SW] (Figure 4G). Together, our data demonstrate a critical in vivo requirement for SEC23B in T cells, and suggest that targeting it may have salutary effects in $\mathrm{T}$ celldependent immune pathologies.

Mechanisms of COPII-dependent regulation of $T$ cell functions. Mammalian cells express 2 paralogs of SEC23, SEC23B and SEC23A $(6,25)$. Recent studies suggest that they are functionally complementary during development and have indistinguishable interactomes (26), but are expressed in a tissue- and species-specific manner lending to distinct phenotypes in mice and humans when deficient or mutated (27-30). Intriguingly, we observed that WT murine T cells harbor both paralogs, and that SEC23B-deficient $\mathrm{T}$ cells maintained normal expression of SEC23A (Figure 1C). Despite the presence of SEC23A, however, the absence of SEC23B critically impacted $\mathrm{T}$ cell functions. We therefore next determined the mechanisms leading to the $\mathrm{T}$ cell defects despite the presence of normal SEC23A levels in SEC23B-deficient T cells. Specifically, we considered 2 mutually exclusive hypotheses: (a) SEC23B possesses independent functions that are not compensated for by SEC23A, or (b) because mature T cells express both SEC23B and SEC23A, the functional effect of $\operatorname{Sec} 23 b$ deletion is from a net loss of total SEC23. If the latter, we reasoned that increased expression of SEC23A, in the absence of SEC23B, to a total level of SEC23 similar to that present in WT would rescue T cell functions.

To explore this, we first generated $\operatorname{Sec} 23 a^{f l-} C d 4^{C r e}$ mice, which lack SEC23A specifically in $\mathrm{T}$ cells but harbor normal levels of SEC23B (Figure 5A). SEC23A-deficient T cells developed normally and in the usual proportions (Supplemental Figure 3). In the absence of SEC23A, T cells demonstrated normal IL-2 secretion 
A
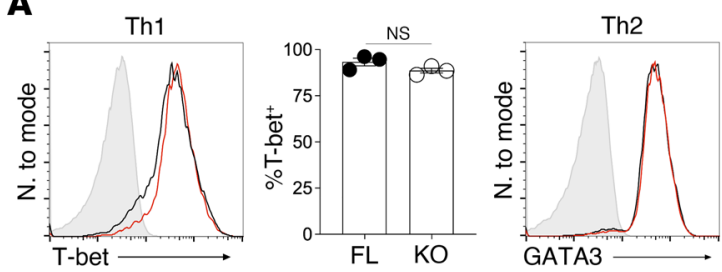

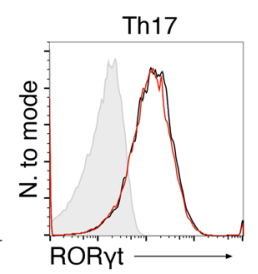

FL KO

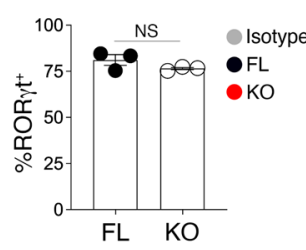

C

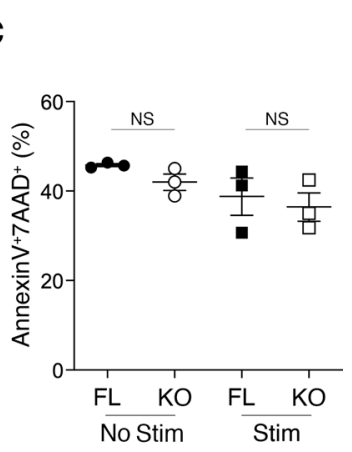

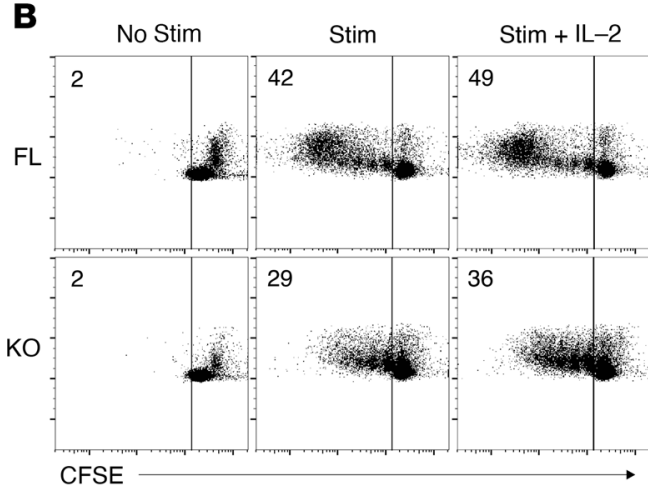

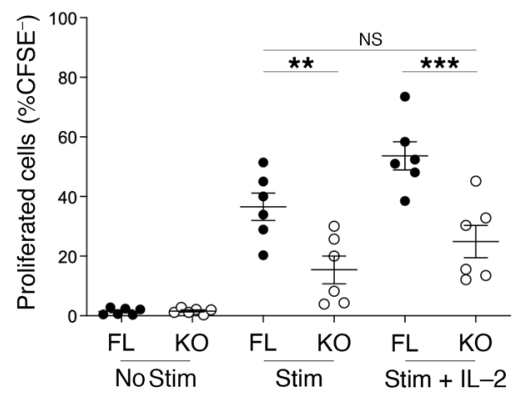

D

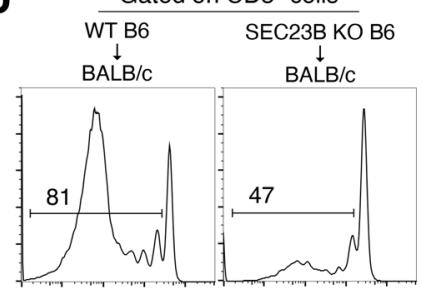

CFSE

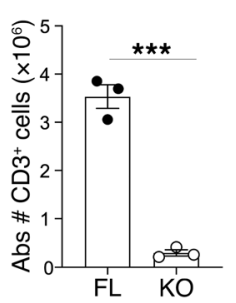

E

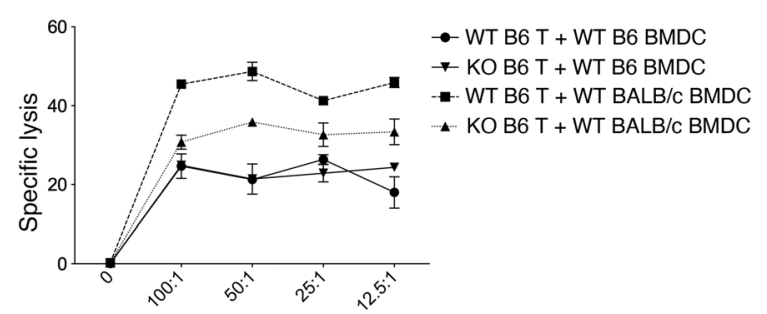

$\mathbf{F}$
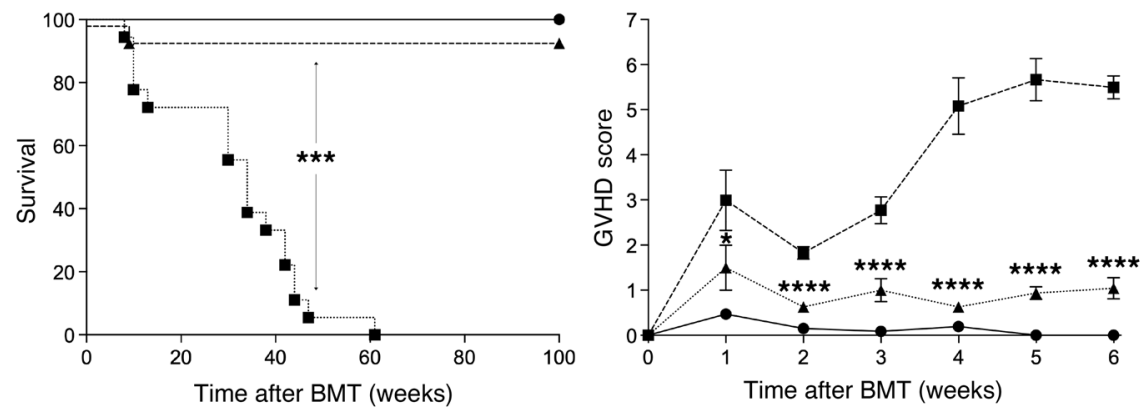

G
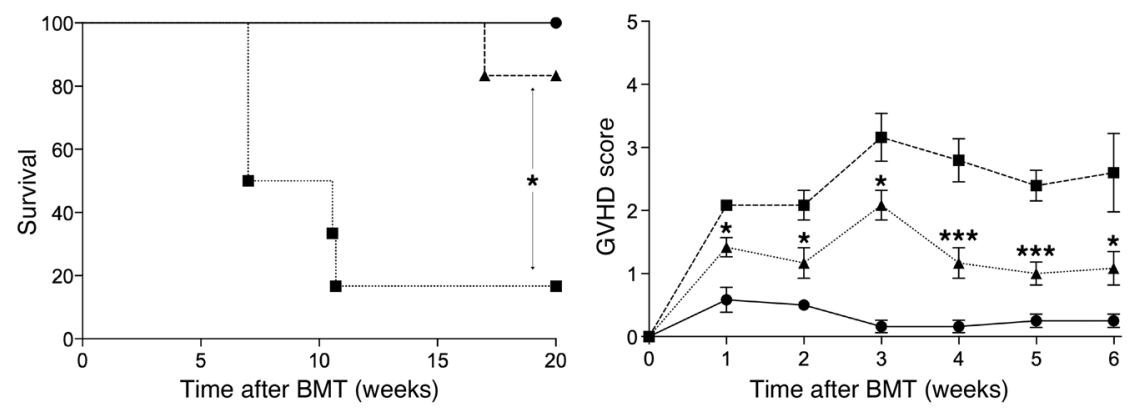

$\rightarrow$ WT B $6 \rightarrow$ WT B 6

- WT B6 $\rightarrow$ WT C3H.SW $\cdots$ - … SEC23B KO B6 $\rightarrow$ WT C3H.SW 
Figure 4. SEC23B-dependent COPII regulates naive T cell functions in vitro and in vivo. (A) Overlay of histograms based on flow cytometry plots indicating expression of intracellular T-bet, GATA3, and ROR $\gamma \mathrm{t}$ in WT and SEC23B-deficient naive CD4 ${ }^{+}$T cells that were cultured in media containing Th1-, Th2-, and Th17-polarizing cytokines, respectively $(n=3)$. (B) Proliferative capacity of WT and SEC23B-deficient T cells as measured by CFSE dilutions in vitro ( $n=6$ /group). Partial rescue of proliferation upon addition of exogenous murine IL- 2 in T cells stimulated with $\alpha$ CD3 and $\alpha$ CD28 for 3 days ( $n=6 /$ group). (C) Cell death as measured by Annexin $V$ and 7AAD staining ( $n=3 /$ group) in SEC23B-deficient T cells compared with WT following stimulation by $\alpha \mathrm{CD} 3$ and $\alpha \mathrm{CD} 28$ for 3 days. (D) In vivo proliferative capacity of SEC23B-deficient T cells compared with WT as measured by CFSE dilutions following their transfer into allogeneic BALB/C recipient mice ( $n=3 /$ group). (E) ${ }^{51} \mathrm{Cr}$ release assay using WT or SEC23Bdeficient T cells primed with allogeneic splenic cells and used as effector cells against either syngeneic $[57 \mathrm{BL} / 6$ ) or allogeneic BALB/C BMDC target cells ( $n=3$ /group). (A, C and D) Statistical significance was determined by 2-tailed unpaired Student's $t$ test; ${ }^{* *} P<0.001$. (B) Statistical significance was determined by 1-way ANOVA and post hoc Tukey's test; ${ }^{* *} P<0.01$, ${ }^{* *} P<0.001$. (F and $\left.\mathbf{C}\right)$ Survival and GVHD scores of mice that received syngeneic $T$ cell-depleted bone marrow, and T cells from either syngeneic donors, allogeneic WT C57BL/6) donors, or allogeneic Sec23b $b^{f /-}$ Cd4 ${ }^{\text {Cre }}$ C57BL/6) donors. Allogeneic recipients were BALB/C ( $n=12 /$ group) $(\mathbf{F})$ or C3H.SW ( $n=6 /$ group) (G) strain. Significance was determined by 1-way ANOVA and post hoc Tukey's test for GVHD score and Mantel-Cox log rank test for survival; ${ }^{*} P<0.05,{ }^{* *} P<0.01,{ }^{* * *} P<0.001$, ${ }^{* * *} P<0.0001$.

(Figure 5B), no abnormal increase in accumulation of intracellular IL-2 (Figure 5C), and similar proliferation following activation when compared with WT (Figure 5D). These data suggested that SEC23B, which is present at greater baseline levels in T cells, functionally compensates for loss of SEC23A, indicating that either SEC23A is dispensable in T cells or that T cells are sensitive to levels of total available SEC23 protein.

To directly test this, we next analyzed $\operatorname{Sec} 23^{b-a / b-a}$ mice, which lack $\operatorname{Sec} 23 b$ but express $\operatorname{Sec} 23 a$ from the endogenous $S e c 23 b$ genomic locus (Figure 5E) and thus produce additional SEC23A, but only at a level comparable to the total sum of wild-type SEC23A and SEC23B (26). We confirmed that $S e c 23^{b-a / b-a}$ T cells developed normally (Supplemental Figure 3) and lacked SEC23B, but contained increased levels of SEC23A (Figure 5A). Following activation, Sec $23^{b-a / b-a}$ T cells exhibited normal proliferative capacity (Figure 5D). These findings thus demonstrate that SEC23A and SEC23B have overlapping functions, and that T cells depend on total levels of COPII protein SEC23 for their secretory and effector functions in vitro.

To definitively confirm that SEC23A can compensate for SEC23B when expressed at sufficient levels in vivo, we performed allo-HCT to determine the pathogenic capacity of SEC23A-deficient T cells and $\operatorname{Sec} 23^{b-a / b-a} \mathrm{~T}$ cells. We hypothesized that in contrast to SEC23B-deficient T cells, SEC23A-deficient T cells and $\operatorname{Sec} 23^{b-a / b-a} \mathrm{~T}$ cells would induce full GVHD severity. All control recipients received a dose of WT C57BL/6J bone marrow and syngeneic T cells. Allogeneic recipients received WT C57BL/6J bone marrow and either allogeneic WT C57BL/6J T cells, SEC23A-deficient $\mathrm{T}$ cells, or Sec23 $3^{b-a / b-a} \mathrm{~T}$ cells. Consistent with our hypothesis, all recipients of allogeneic $\operatorname{Sec} 23^{b-a / b-a} \mathrm{~T}$ cells expired (Figure $5 \mathrm{~F}$ ), and survival was similar in recipients of $\operatorname{Sec} 23^{b-a / b-a} \mathrm{~T}$ cells as those of WT T cells. Recipients of SEC23A-deficient T cells also expired due to GVHD, although duration of survival was moder- ately improved when compared with recipients of WT T cells, possibly due to reduced overall levels of SEC23 (Figure 5F). Transfer of $\operatorname{Sec} 23^{b-a / b-a} \mathrm{~T}$ cells also resulted in similar GVHD clinical scores when compared with WT T cells, with recipients of SEC23A-deficient $\mathrm{T}$ cells displaying clinical scores of intermediate severity (Figure 5F). These data further demonstrate that while SEC23B is the predominant paralog in T cells, SEC23A can compensate for its loss in a dose-dependent manner in vivo.

Characterizing the role of SEC23 paralogs in human $T$ cells. Homozygous or compound heterozygous mutations in SEC23B give rise to CDAII in humans $(18,25)$. Patients with CDAII exhibit a mild to moderate anemia primarily due to ineffective erythropoiesis and other red blood cell abnormalities $(25,31)$. However, this phenotype is not observed in mice (27-29). While CDAII manifests predominantly as a disorder of erythrocytes, reduced SEC23B has been documented in lymphocytes in patients with CDAII compared with healthy controls (32). We therefore first explored the relevance of the SEC23B-dependent COPII pathway in normal human $\mathrm{T}$ cell functions in vitro. Similarly to WT mice, healthy human T cells expressed both SEC23A and SEC23B (Figure 6A). We therefore used CRISPR/Cas9 via nucleofection of Cas9:guide RNA ribonucleoprotein to knock-out Sec23b in T cells obtained from healthy donors (Figure 6B). Following nucleofection, we confirmed a reduction in SEC23B expression by quantitative reverse transcription PCR (qRT-PCR) and Western blot (Figure 6C), and assessed $\mathrm{T}$ cell functions. We observed that healthy human T cells that received $\operatorname{Sec} 23 b$-targeting guide RNAs exhibited reduced IL-2 secretion (Figure 6D) and increased IL-2 retention as measured by intracellular flow cytometry (Figure $6 \mathrm{E})$ when compared with $\mathrm{T}$ cells receiving nontargeting controls. We next performed xenogeneic bone marrow transplantation of either control or CRISPR/Cas9-mediated Sec23b KO human T cells into NOD-scid-IL2R $\gamma$ (NSG) recipient mice. We hypothesized that mice receiving $\operatorname{Sec} 23 b \mathrm{KO}$ T cells would exhibit decreased GVHD severity and greater survival when compared with recipients receiving control $\mathrm{T}$ cells. Consistent with this, all NSG mice transplanted with control $\mathrm{T}$ cells expired. However, $60 \%$ of mice that received KO T cells survived by 35 days, and also exhibited lower posttransplant GVHD scores (Figure 6F).

Together, these data suggested that patients with CDAII who are deficient in SEC23B will also exhibit T cell defects. Therefore, we next analyzed $\mathrm{T}$ cells obtained from a patient with CDAII. We first activated the patient's T cells for 3 days with $\alpha \mathrm{CD} 3$ and $\alpha \mathrm{CD} 28$, and measured their proliferation and cytokine secretion. In contrast to murine SEC23B-deficient $T$ cells and healthy human $\mathrm{T}$ cells that underwent acute knock-down of Sec23b, T cells from the patient with CDAII exhibited normal proliferative capacity (Figure 6G) and appeared to produce and release IL-2 normally, as well as TNF- $\alpha$ and IFN- $\gamma$ (Figure 6H). The patient's T cells also expressed levels of surface CD69 and CD25 similar to those in healthy controls (Figure 6I). Because mechanistic murine studies demonstrated that SEC23A can rescue cytokine secretion in the absence of SEC23B when expressed in sufficient quantities, we hypothesized that preservation of the $\mathrm{T}$ cell response by the patient with CDAII may be from a compensatory increase in SEC23A as in our murine studies from above. To test this, we measured SEC23A levels in T cells from 3 patients with CDAII. We found that SEC23A 
A
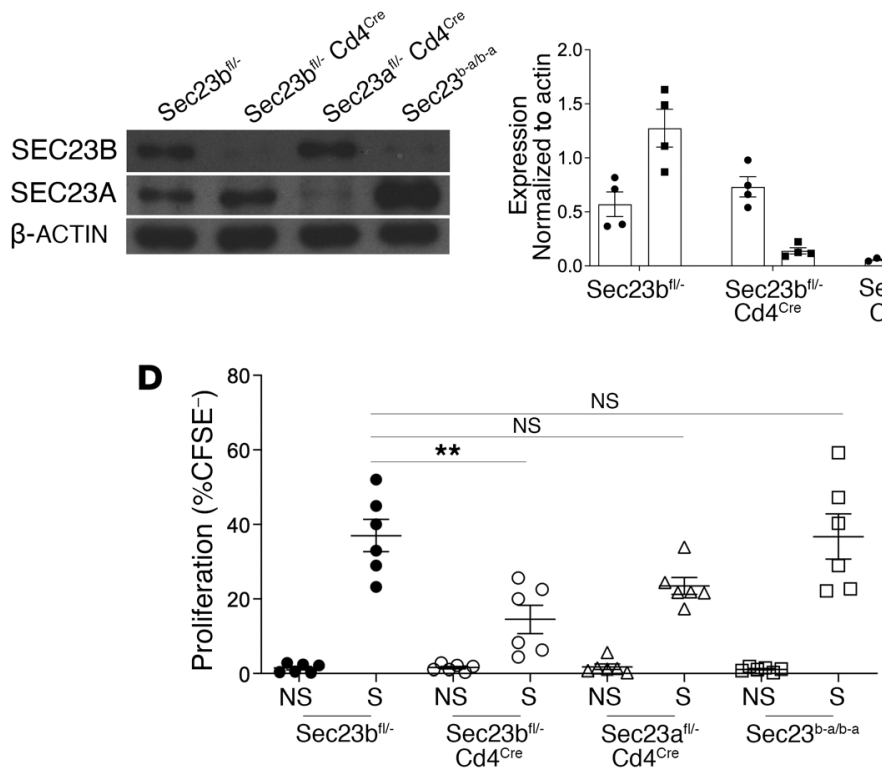

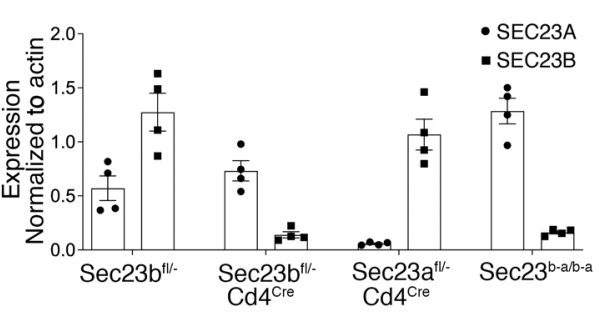

B

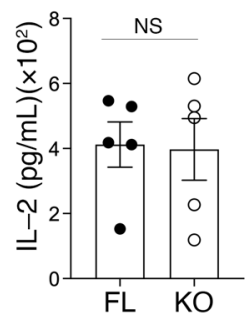

C

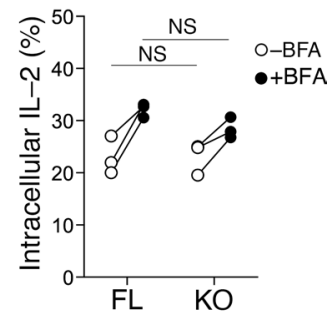

E

Sec23b

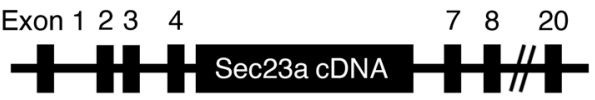

$\mathbf{F}$
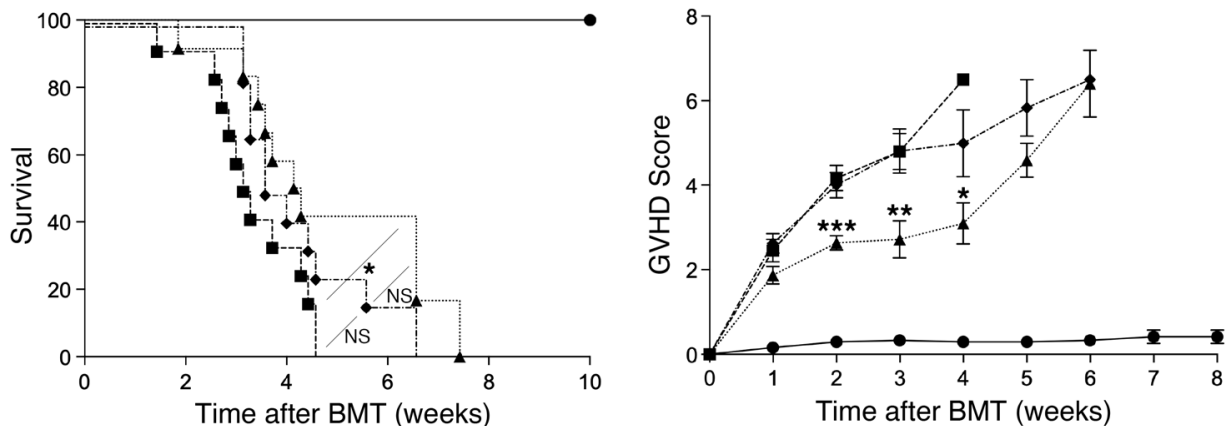

- WT B6 $\rightarrow$ WT B6

$\cdots-$ - SEC23A KO B6 $\rightarrow$ WT BALB/C

WT B6 $\rightarrow$ WT BALB/C

SEC23 ${ }^{b-a b-a} B 6 \rightarrow W T$ BALB/C

Figure 5. Mechanisms of COPII-dependent regulation of T cell functions. (A) Representative Western blot showing SEC23B and SEC23A expression in purified T cells from $\operatorname{Sec} 23 b^{f / /}, \operatorname{Sec} 23 b^{f / /-} C d 4^{c r e}$, Sec $23 a^{f / /}-C d 4^{c r e}$, and $\operatorname{Sec} 23^{b-a / b-a}$ mice. Normalized levels of each protein relative to $\beta$-actin are plotted on the right $(n=$ 4/group). (B) ELISA measuring IL-2 secreted by WT and SEC23A-deficient T cells over 3 days in culture with $\alpha$ CD3- and $\alpha$ CD28-stimulating antibodies ( $n=5 /$ group). (C) Flow cytometry measuring levels of intracellular IL-2 in WT and SEC23A-deficient T cells following their stimulation in vitro for 3 days with $\alpha$ CD3 and $\alpha \mathrm{CD} 28$, and 5 hours with PMA and ionomycin in the presence or absence of BFA ( $n=3 / g r o u p)$. (D) Proliferation of isolated T cells from the indicated mice stimulated in vitro with $\alpha \mathrm{CD} 3$ and $\alpha \mathrm{CD} 28$, as measured by CFSE dilutions ( $n=6 / \mathrm{group}$ ). (E) Schematic diagram of Sec23b endogenous genomic locus in $\operatorname{Sec} 23^{b-a / b-a}$ mice, which contains the Sec23a coding sequence (from C367 to A2298) followed by a poly(A) termination signal in lieu of the full Sec $23 b$ sequence as previously described (26). (F) Survival and composite GVHD scores of mice ( $n=12$ /group) that received syngeneic T cell-depleted bone marrow and T cells from either syngeneic WT C57BL/6) donors or allogeneic WT C57BL/6], Sec23a fl/- Cd4 ${ }^{\text {rre }}$ C57BL/6], or Sec23b-a/b-a C57BL/6) donors. (B and C) Significance was determined by 2 -tailed unpaired Student's $t$ test. (D) Significance was determined by 1-way ANOVA and post hoc Tukey's tests; ${ }^{* *} P<0.01$. (F) Significance was determined by Mantel-Cox log rank test for survival and 1-way ANOVA and post hoc Tukey's tests for GVHD score; ${ }^{*} P<0.05,{ }^{* *} P<0.01,{ }^{* * *} P<0.001$.

was expressed in all 3 patients at about twice the levels observed in healthy controls (Figure 6J). Altogether, these data confirmed the interchangeability of SEC23 paralogs in T cells from mice and humans, and also provided a potential explanation for the lack of significant immunodeficiencies in patients with CDAII $(33,34)$.

\section{Discussion}

Most COPII subunits in mammals exist in multiple paralogs, and mutations in different paralogs often lead to distinct disease phenotypes $(4,25)$. Previous studies have proposed that SEC23A and SEC23B may contribute to increased complexity of COPII by car- rying out nonoverlapping functions $(10,11,35)$. Recent studies that have shown that mice and humans have distinct phenotypes when they lack SEC23B also support the hypothesis that tissue-specific patterns of $\operatorname{Sec} 23 a$ or $\operatorname{Sec} 23 b$ expression directed by evolutionary shifts in gene expression contribute to these differences (27-29). This has been supported by findings that SEC23A and SEC23B have indistinguishable interactomes and are interchangeable during development in vivo (26). In contrast to these studies, our study demonstrates that $\mathrm{T}$ cells derived from healthy mice and humans express both SEC23B and SEC23A. Importantly, while $\mathrm{T}$ cells from patients with CDAII exhibit increased expression 
of SEC23A, knockdown of Sec23b in mature T cells from healthy humans did not upregulate SEC23A, presumably due to the acute nature of knock-down by CRISPR/Cas9 in T cells and transcriptional regulation of SEC23 expression during hematopoietic cell development. The functions of T cells from the patient with CDAII are comparable to those derived from healthy controls in their ability to become activated, proliferate, and secrete cytokines. These observations may be due to differences in genetic compensation mechanisms in human $\mathrm{T}$ cells that manifest in gene knock-out when compared with knockdown methods (36). Although poorly understood, functional compensation by paralogs is a common phenomenon in mouse KO studies (37), and the regulated expression of a protein by its paralog has been observed in $\mathrm{T}$ cells in other contexts (38). Our observations thus extend our understanding of the role of paralog specificity in COPII-mediated transport in vivo, while demonstrating for what we believe is the first time a role for this intracellular pathway in $\mathrm{T}$ cell immunity.

The intracellular pathways and their molecular determinants that regulate $\mathrm{T}$ cell secretion remain poorly characterized. Using genetically modified mice, we show that $\operatorname{Sec} 23 b$ mutation significantly alters the $\mathrm{T}$ cell secretome and impairs the secretion of multiple proteins. Notably, we observed that the secretion of important T cell-derived factors including IL-2 is dependent on levels of available SEC23, and that they are retained intracellularly when SEC23 is reduced. The secretion of inflammatory cytokines by $\mathrm{T}$ cells is central to the pathogenesis of multiple diseases. Our findings suggest that the reduction of SEC23-dependent COPII formation is sufficient to substantially reduce morbidity and mortality related to GVHD in mice without affecting $\mathrm{T}$ cell development. Our observations suggesting that the secretion defect is not global, but rather impacts COPII-dependent secretory cargoes, may present an opportunity to target SEC23 in COPII as a novel strategy to mitigate T cell-mediated pathologies such as GVHD. Conversely, it is possible that enhancing COPII may increase $\mathrm{T}$ cell function as a therapeutic approach to other disorders. The differences in cytokine secretion patterns we observed may be due to differences the ability of these cytokines to utilize alternate secretory pathways or differentially interact with cargo adapters. While several known COPII cargoes have been identified in other cell types, the differential regulation of cytokine secretion by COPII is not well understood.

A large number of proteins are transported via the COPII pathway, including integral membrane proteins, secreted proteins, and those destined for other intracellular compartments including the nucleus. For example, transcription factors such as SREBPs are known to be dependent on COPII for their activity $(39,40)$, and also regulate $\mathrm{T}$ cell functions (41). When we supplemented our $\mathrm{T}$ cell cultures with exogenous IL-2, T cell proliferation was rescued only partially, raising the likelihood that other $\mathrm{T}$ cell processes are also affected by $\operatorname{Sec} 23 \mathrm{~b}$ deletion and contribute to its overall functional impact. Therefore, the net functional effect of disrupting $S e c 23 b$ expression is likely a result of a reduction in the extracellular transport of effector molecules and growth factors such as but not limited to IL-2, and additionally include the intracellular transport of other proteins such as transcription factors. Further studies are needed to elucidate the role of COPII-mediated intracellular transport of transcription factors in addition to secreted proteins in regulating $\mathrm{T}$ cell functions. It is formally possible that SEC23 functions independent of COPII formation may also contribute to the observed phenotype; however, no functions outside of COPII have been attributed to SEC23. Finally, in this study, we focused on a critical role for SEC23B-dependent COPII on the functions of naive T cells. Whether this fundamental pathway affects other immune cell subsets, including innate immune cells, will be determined in future studies.

\section{Methods}

Mice. The generation of $\operatorname{Sec} 23 b^{f /-}, \operatorname{Sec} 23 a^{f /-}$, and $\operatorname{Sec} 23^{b-a / b-a}$ mice has been previously reported by us (26-28). $\operatorname{Sec} 23 b^{f /-}$ and $\operatorname{Sec} 23 a^{f /-}$ mice were bred with $C d 4^{\text {Cre }}$ (catalog 017336) or Vav1 ${ }^{\text {Cre }}$ (catalog 008610) mice obtained from The Jackson Laboratory. Genotyping was performed using primers previously described (26-28). C57BL/6C (strain code 027 ) and BALB/c (strain code 028) mice used as recipients in BMT experiments were obtained from Charles River Laboratories, and C3H.SW (catalog 000438) and NSG (catalog 005557) recipient mice were obtained from The Jackson Laboratory. NSG mice were placed in animal biosafety level 2 containment housing throughout the duration of the experiment described.

$T$ cell isolation and in vitro cell culture. Primary murine T cells were purified by magnetic separation (Miltenyi Biotec) from single cell homogenates of spleens and lymph nodes, and cultured in cell media (1640 RPMI supplemented with 10\% heat inactivated FBS, $2 \mathrm{mM}$ L-glutamine, penicillin-streptomycin $100 \mathrm{U} / \mathrm{mL}, 100 \mathrm{mM}$ HEPES, nonessential amino acids, $1 \mathrm{mM}$ sodium pyruvate, and $50 \mu \mathrm{M} \beta$-mercaptoethanol). T cells were cultured alone, or with $5 \mu \mathrm{g} / \mathrm{mL} \alpha \mathrm{CD} 3$ (clone 145-2C11, BioLegend) and $2.5 \mu \mathrm{g} / \mathrm{mL} \alpha \mathrm{CD} 28$ (clone 37.51, BioLegend) soluble antibodies for the specified time points. For proliferation studies, $\mathrm{T}$ cells were first labeled on day 0 with $5 \mu \mathrm{M}$ CFSE (Thermo Fisher Scientific) for 10 minutes at $37^{\circ} \mathrm{C}$. Prior to analysis of cytokine production by intracellular flow cytometry, $\mathrm{T}$ cells were additionally stimulated on day 3 with PMA and ionomycin (Invitrogen) in the presence or absence of BFA (BioLegend) for 5 hours at $37^{\circ} \mathrm{C}$.

Flow cytometric analysis. Flow cytometric analysis was performed using fluorophore-conjugated antibodies to mouse CD3 (clone 1452C11), CD4 (clone GK1.5), CD8 (clone 53-6.7), CD69 (clone H1.2F3), CD25 (clone 3C7), IL-2 (clone JES6-5H4), IFN- $\gamma$ (clone XMG1.2), TCR- $\beta$ (clone H57-597), Annexin V, and 7-AAD (catalog 640922) (BioLegend). For surface immunophenotyping, single-cell suspensions were incubated with the relevant antibodies for 30 minutes at $4^{\circ} \mathrm{C}$, washed, and fixed with $1 \%$ paraformaldehyde prior to analysis. Intracellular staining was performed following surface staining and fixation, in permeabilization buffer (eBioscience) for 30 minutes at room temperature. Staining of phosphorylated proteins was performed on methanol-fixed cells permeabilized with True-Phos Perm Buffer (BioLegend) according to the manufacturer's instructions. All samples were run on an Attune NxT flow cytometer and analysis was performed using FlowJo v10.3.

T cell immunofluorescence confocal microscopy. Purified T cells were fixed on coverslips with 3\% paraformaldehyde in PBS, and quenched with $50 \mathrm{mM} \mathrm{NH}_{4} \mathrm{Cl}$ in PBS. Cells were then permeabilized in $0.3 \%$ Triton X-100 in PBS and blocked with 10\% normal goat serum. Cells were then sequentially stained with IL-2 (clone JES6-5H4, BioLegend) and SEC23B (catalog PA5-52533, Invitrogen) followed by staining with

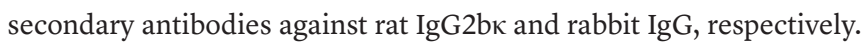
DAPI (Life Technologies) was used to detect nuclei. Acquisition of 
A

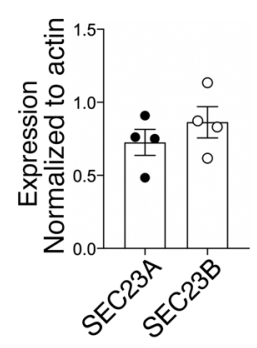

C

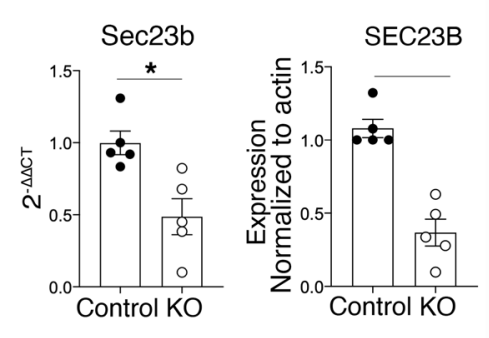

$\mathbf{F}$

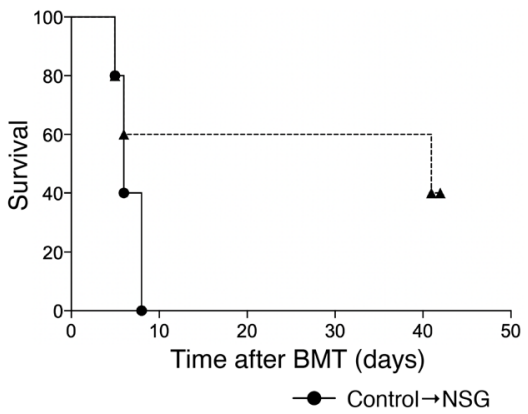

G

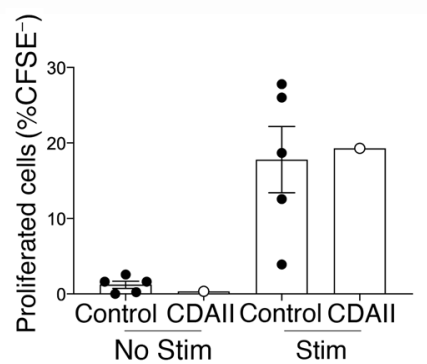

B

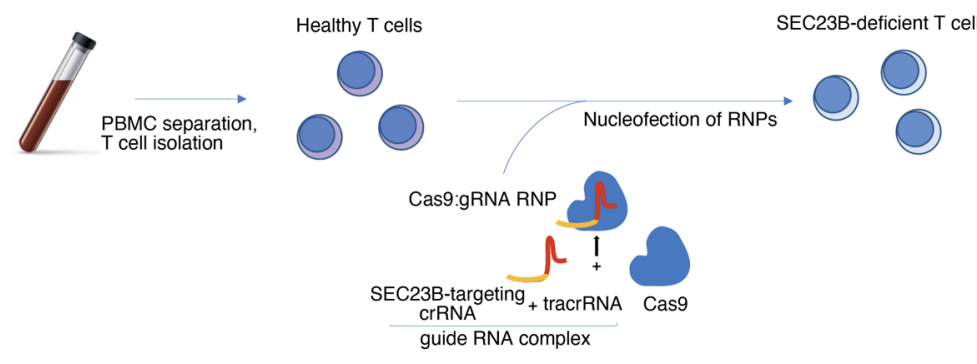

D

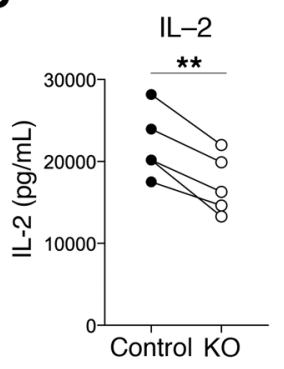

E

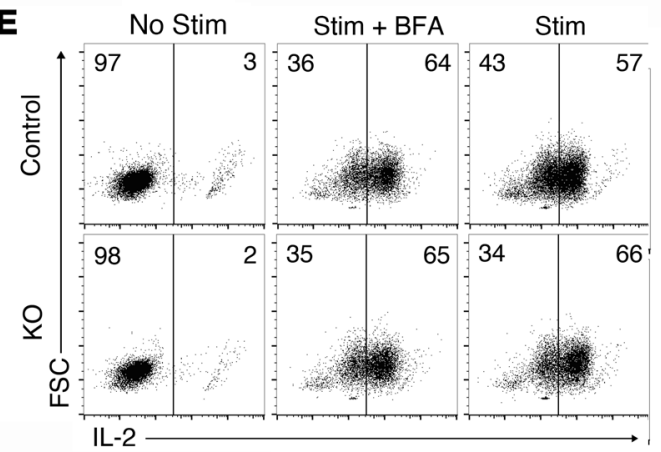

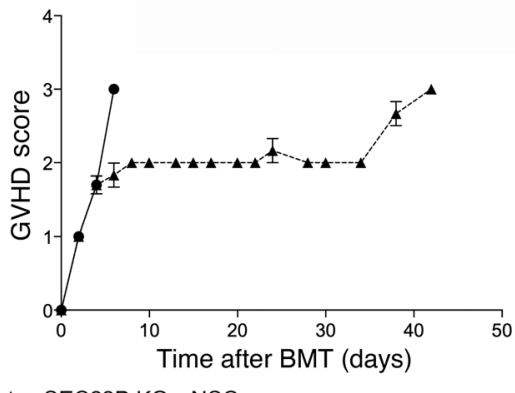

- SEC23B KO $\rightarrow$ NSG

H
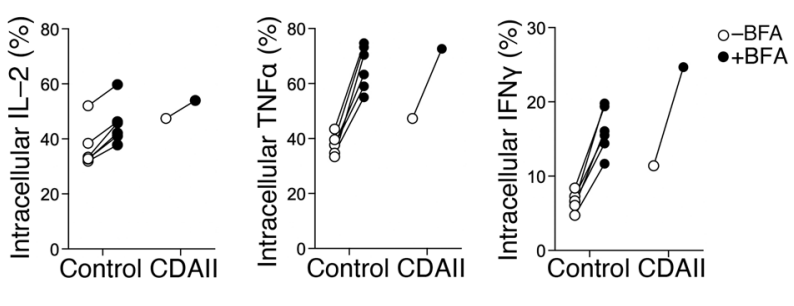

I
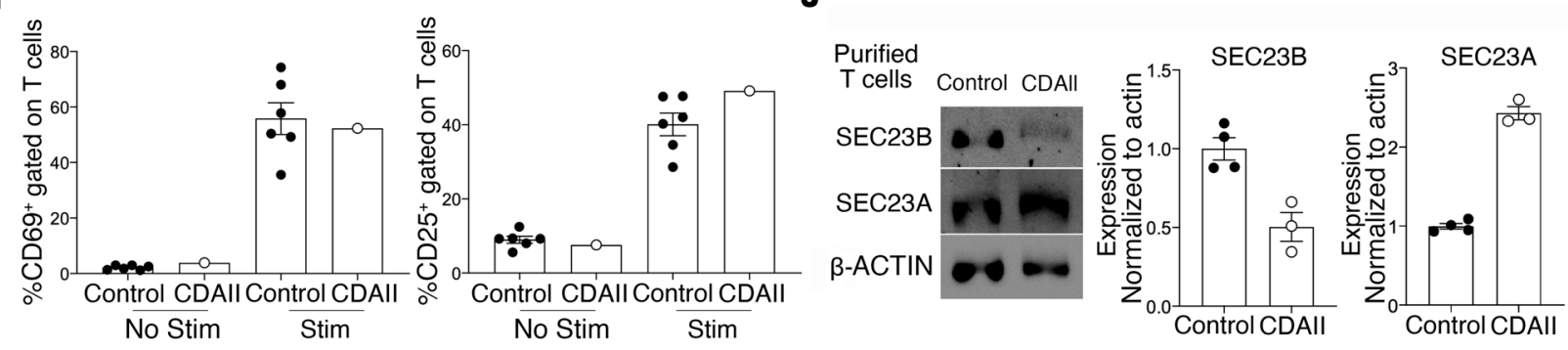
Figure 6. Characterizing the role of SEC23 paralogs in human T cells. (A) Normalized expression levels of SEC23B and SEC23A in naive T cells isolated from healthy humans relative to $\beta$-actin by Western blot $(n=4 /$ group). (B) Experimental steps in CRISPR/Cas9-mediated KO by Cas9/RNP nucleofection in healthy human T cells. (C) qRT-PCR and Western blot analysis of knock-out efficiency of T cells that underwent CRISPR/Cas9-mediated Sec23b KO. T cells were analyzed 3 days following nucleofection with control or Sec23b-targeting crRNA-tracrRNA duplexes complexed with Cas 9 ( $n=5$ /group). (D) ELISA measuring IL-2 secreted by control or KO T cells ( $n=5$ /group). (C and D) Data represent mean \pm SEM, with ${ }^{*} P<0.05$ and ${ }^{*} P<0.01$ (2-tailed unpaired Student's $t$ test). (E) Representative flow cytometric plots demonstrating intracellular IL-2 in healthy T cells that underwent CRISPR/Cas9-based Sec23b KO compared with those that received control guide RNA. (F) Survival curve and CVHD scores of NSC mice that received control or CRISPR/Cas9-mediated SEC23B KO T cells $(n=5$ / group). (C) Proliferative capacity of T cells derived from a patient with CDAll compared with healthy controls, as measured by CFSE dilutions following stimulation in vitro with $\alpha \mathrm{CD} 3$ and $\alpha \mathrm{CD} 28$ for 3 days ( $n=5 /$ controls, $n=1$ / CDAII). (H) Flow cytometry measuring levels of intracellular IL-2, TNF- $\alpha$, and IFN- $\gamma$ in T cells from a patient with CDAll and healthy controls, following stimulation in vitro with $\alpha \mathrm{CD} 3$ and $\alpha \mathrm{CD} 28$ for 3 days, and an additional 5 hours with PMA and ionomycin in the presence or absence of BFA ( $n=5 /$ controls, $n=1 /$ CDAII). (I) Quantification of surface CD69 and CD25 expression as measured by flow cytometry in T cells from healthy controls or from a patient with CDAll on day 3 after no stimulation or stimulation with $\alpha \mathrm{CD} 3$ and $\alpha \operatorname{CD28}(n=6 /$ controls, $n=1 /$ CDAll). (J) Western blots showing expression of SEC23B and SEC23A relative to $\beta$-actin in naive unmanipulated T cells from healthy human donors or patients with CDAll ( $n=4 /$ controls, $n=3 /$ CDAll).

images was performed in a blinded manner using a Nikon A1 inverted point-scanning confocal microscope with standard PMT detectors.

Quantitative RT-PCR. RNA was isolated from T cells with the Qiagen RNeasy kit. cDNA was synthesized using the High Capacity cDNA Reverse Transcription Kit (Applied Biosystems), and quantified on a NanoDrop Lite Spectrophotometer (Thermo Fisher Scientific). Quantitative RT-PCR was performed using PowerUP SYBR Green Master Mix (Applied Biosystems) and the primers listed in Supplemental Table 1 on a Mastercycler RealPlex ${ }^{2}$ (Eppendorf).

Western blotting. Whole-cell lysates obtained from purified $\mathrm{T}$ cells were fractionated by gel electrophoresis through a $10 \%$ BisTris gel and transferred onto polyvinylidene difluoride membrane (Millipore) in a semi-dry transfer cell (Bio-Rad). Blots were blocked with $5 \%$ nonfat milk in TBST, stained with primary antibody overnight at $4^{\circ} \mathrm{C}$, washed with TBST, and stained with secondary antibody for 2 hours at room temperature. The following primary antibodies were used: rabbit anti-SEC23B (LTKSAMPVQQARPAQPQEQP), rabbit anti-SEC23A (DNAKYVKKGTKHFEA) (8), and mouse anti- $\beta$-Actin (catalog 8226, Abcam). Secondary antibodies anti-rabbit IgG conjugated to HRP (catalog sc-2357) and mouse IgG kappa biding protein-HRP (sc-516102; Santa Cruz Biotechnology) were used to detect primary antibodies. Blots were detected using the SuperSignal ECL substrate system (Thermo Fisher Scientific), on a Konica SRX-101A film processor. Densitometric analysis was performed with Image J software. See complete unedited blots in the supplemental material.

Enzyme-linked immunosorbent assay. Supernatants from cell culture were harvested at the specified time points and analyzed for levels of IL-2 (catalog 555240, BD Biosciences), IFN- $\gamma$ (catalog 555240, BD Biosciences), and TNF- $\alpha$ (catalog MTAOOB, R\&D Systems) by ELISA. Measurement of bulk cytokines was performed using a 29-multiplex array (Millipore Sigma) according to the manufacturer's instructions on the Luminex 200 IS analyzer (Affymetrix) at the University of Michigan Cancer Center Immunology Core.

${ }^{51}$ Chromium release assay. $\mathrm{CD} 8^{+} \mathrm{T}$ cells were purified by magnetic separation (Miltenyi Biotec), stimulated by irradiated (300 Gy) allogeneic splenic APCs, and used as effector cells. BMDCs derived from $\mathrm{BALB} / \mathrm{c}$ bone marrow were harvested after 7 days of cell culture in GM-CSF (20 ng/mL, PeproTech, catalog 315-03) containing media, labeled by incubation with $2 \mathrm{MBq}$ of $\mathrm{Na}_{2}{ }^{51} \mathrm{CrO}_{4}$ (PerkinElmer Life) for 2 hours at $37^{\circ} \mathrm{C}$ in $5 \% \mathrm{CO}_{2}$, and used as target cells. Labeled target cells were plated with effector cells in triplicate at varying effector-to-target ratios, and incubated for 4 hours. Maximal and minimum release was determined by the addition of Triton-X (MP Biomedicals) or media alone to targets, respectively. Supernatants were transferred to a Luma plate (PerkinElmer) after 4 hours, and ${ }^{51} \mathrm{Cr}$ activity was determined using an autogamma counter (Packard).

Bone marrow transplantation and xenogeneic GVHD model. BMTs were performed as previously described $(42,43)$. Briefly, syngeneic $[\mathrm{C} 57 \mathrm{BL} / 6 \mathrm{~J} \rightarrow \mathrm{C} 57 \mathrm{BL} / 6 \mathrm{C}]$ and allogeneic $[\mathrm{C} 57 \mathrm{BL} / 6 \mathrm{~J} \rightarrow \mathrm{BALB} / \mathrm{c}$ or $\mathrm{C} 57 \mathrm{BL} / 6 \mathrm{~J} \rightarrow \mathrm{C} 3 \mathrm{H} . \mathrm{SW}]$ recipients received lethal total body irradiation on day -1 . BALB/c recipients received a total of $8 \mathrm{~Gy}\left({ }^{137} \mathrm{Cs}\right.$, split dose separated by 3 hours), and C57BL/6C and C3H.SW recipients received a single dose of $10 \mathrm{~Gy}\left({ }^{137} \mathrm{Cs}\right.$, single dose $)$. All mice received $1 \times 10^{6} \mathrm{~T}$ cells from the specified donors, and $5 \times 10^{6}$ WT T cell-depleted bone marrow cells. Survival was monitored daily, and recipient GVHD clinical scores were measured weekly according to the severity of disease on recipient weight loss, posture, mobility, skin, and fur integrity as previously described (42). In xenograft GVHD experiments, NSG recipient mice were injected intravenously with human T cells $(1.5 \times$ $\left.10^{6}\right)$ that received either control nontargeting crRNA or Sec $23 b$-targeting crRNA by CRISPR/Cas9 RNP nucleofection as described below. Nucleofection of donor T cells was performed on day -3. NSG mice were irradiated with $200 \mathrm{cGy}\left({ }^{137} \mathrm{Cs}\right.$, single dose) on day -1 , and transplants were performed on day 0 . NSG recipients were maintained on acidic water $(\mathrm{pH}=3.0)$ during the entire course of the experiment. Survival was monitored daily and GVHD clinical scores were assessed 2 to 3 times weekly.

Isolation of human T lymphocytes. PBMCs were isolated from human whole blood by density centrifugation with Ficoll-Paque Premium (GE Healthcare) according to the manufacturer's instructions. PBMCs were treated with red blood cell lysis buffer (MilliporeSigma) and cryopreserved in heat-shocked fetal calf serum (Gibco) containing $10 \%$ DMSO (MilliporeSigma). Prior to use, PBMCs were thawed in a $37^{\circ} \mathrm{C}$ water bath and immediately resuspended in complete cell media supplemented with $50 \mathrm{U} / \mathrm{mL}$ Benzonase (EMDMillipore). T cells were isolated from PBMCs by magnetic separation (Miltenyi Biotec) and used for downstream analysis.

CRISPR/Cas9-mediated knock-out in $T$ cells by ribonucleoprotein (RNP) nucleofection. CRISPR/Cas9 RNP nucleofection was carried out as previously described (44). Briefly, T cells were isolated from healthy human PBMCs by magnetic separation (Miltenyi Biotec) and incubated in media containing IL-7 (10 ng/mL) for 2 to 24 hours $\left(37^{\circ} \mathrm{C}, 5 \%\right.$ $\mathrm{CO}_{2}$ ). Control nontargeting crRNA (Integrated DNA Technologies) or Sec23b-targeting crRNA (AGGTCTGGACGTTCTTTCAA, GACGTTCTTTCAAAGGAGTA, AAGAGACCTTTGCGATCCAC) were complexed with tracrRNA (IDT) at equimolar concentrations and annealed at $95^{\circ} \mathrm{C}$ for 5 minutes. crRNA-tracrRNA duplexes were com- 
plexed with Cas9 protein (IDT) at room temperature for 10 minutes. T cells were resuspended in primary cell nucleofector solution (Lonza) and mixed with Cas9/RNPs for 2 minutes at room temperature. Combined samples were transferred to a nucleofection cuvette, and electroporated on a Lonza $4 \mathrm{D}$ Nucleofector. Nucleofected T cells were incubated in media at $37^{\circ} \mathrm{C}$ for 3 days in the presence of IL-7 $(10 \mathrm{ng} / \mathrm{mL})$. KO efficiency was confirmed by qRT-PCR and Western blot. T cells were used on day 3 for downstream applications.

Collection of $T$ cell secretome samples. Purified T cells were stimulated by plate-bound $\alpha \mathrm{CD} 3(10 \mu \mathrm{g} / \mathrm{mL})$ and soluble $\alpha \mathrm{CD} 28(5 \mu \mathrm{g} / \mathrm{mL})$ in CTS OpTmizer serum free medium $\left(37^{\circ} \mathrm{C}, 5 \% \mathrm{CO}_{2}\right)$. After 24 hours, cultures were centrifuged ( $400 \mathrm{~g}, 10$ minutes). Cells underwent flow cytometric analysis to confirm activation (CD69) and degree of cell death (Annexin V, 7-AAD). Supernatants were passed through a $40 \mu \mathrm{m}$ filter and underwent ultracentrifugation $(120,000 \mathrm{~g}, 90$ minutes) to remove exosomes. Samples were then concentrated using a $3 \mathrm{kDa}$ cutoff ultrafilter (5000 $g$, 40 minutes). Protein concentration was determined by BCA protein assay, and samples were stored at $-70^{\circ} \mathrm{C}$ until use. Protocols for TMT labeling and offline fractionation, liquid chromatography-mass spectrometry analysis (LC-MultiNotch MS3), and data analysis are provided in Supplemental Methods. Accession number PXD015766 in the Proteomics Identifications Database (PRIDE).

Statistics. Experiments were conducted with technical and biological replicates at an appropriate sample size, as estimated by our prior experience. An unpaired 2-tailed Student $t$ test was used for statistical evaluation of data between 2 groups, using a statistical software package (GraphPad Prism). Paired Student $t$ test was used in analysis of healthy donor $\mathrm{T}$ cells that received either control nontargeting or Sec23b-targeting guide RNAs. One-way ANOVA followed by Tukey's test was used to compare multiple groups and GVHD clinical scores. Bars and error bars represent the mean and SEM, respectively. Survival curves were plotted using Kaplan-Meier estimates. The Mantel-Cox log-rank test was used to analyze survival data. $P$ less than 0.05 was considered significant. ${ }^{*} P<0.05,{ }^{* *} P<0.01,{ }^{* *} P<0.001,{ }^{* * * *} P<0.0001$. Sample sizes and statistical tests used are detailed in each figure legend.

Study approval. All animal work was performed in accordance with the recommendations of the IACUC of the University of Mich- igan. Human blood sample acquisition and analysis were approved by the Health Sciences and Behavioral Sciences IRB of the University of Michigan (study ID HUM00085214), and the IRB of Cincinnati Children's Hospital (2016-2727). Two patients with a diagnosis of CDAII based on bone marrow studies and/or genetic evaluation were enrolled in CDAR (ClinicalTrials.gov identifier NCT02964494), a registry for patients with the diagnosis of CDA in North America. Written informed consent was obtained from each patient, and analyses of human data were carried out in compliance with ethical regulations.

\section{Author contributions}

SK and PR designed the study. SK, RK, LL, MM, JW, DP, HF, YS, KOW, and RAK performed experiments. RK, TAK, and DG provided critical reagents and expertise. SK, RK, DG, and PR analyzed and interpreted data. SK and PR wrote and edited the manuscript with input from all coauthors.

\section{Acknowledgments}

This work was supported by the National Institutes of Health (grants T32GM007863 and F30AI145113 to SK; grant R01HL148333 to RK; grant R35HL135793 to DG; NHLBI grant R01HL128046 and NCI grants R01CA203542 and R01CA217156 to PR). DG is a Howard Hughes Medical Institute Investigator. We would like to thank the University of Michigan Microscopy and Image Analysis Laboratory for processing and acquisition of TEM samples and for the use of instruments for the acquisition of immunofluorescence confocal images. We would also like to thank the University of Michigan Cancer Center Immunology Core for assistance with Luminex Assays, and the University of Michigan Proteomics Resource Facility for processing and analysis of secretome samples.

Address correspondence to: Pavan Reddy, Department of Internal Medicine, Division of Hematology and Oncology, Blood and Marrow Transplantation Program, University of Michigan Rogel Cancer Center, 7215 CCGC, 1500 E. Medical Center Drive, Ann Arbor, Michigan 48105, USA. Phone: 734.647.5954; Email: reddypr@umich.edu.
1. Barlowe C, et al. COPII: a membrane coat formed by Sec proteins that drive vesicle budding from the endoplasmic reticulum. Cell. 1994;77(6):895-907.

2. Matsuoka $\mathrm{K}$, et al. Coat assembly directs v-SNARE concentration into synthetic COPII vesicles. Mol Cell. 1998;2(5):703-708.

3. Matsuoka K, et al. COPII-coated vesicle formation reconstituted with purified coat proteins and chemically defined liposomes. Cell. 1998;93(2):263-275.

4. Zanetti G, et al. COPII and the regulation of protein sorting in mammals. Nat Cell Biol. 2011;14(1):20-28.

5. Schlacht A, Dacks JB. Unexpected ancient paralogs and an evolutionary model for the COPII coat complex. Genome Biol Evol. 2015;7(4):1098-1109.

6. Fromme JC, et al. Coordination of COPII vesicle trafficking by Sec23. Trends Cell Biol. 2008;18(7):330-336.

7. Yoshihisa T, et al. Requirement for a GTPase-activating protein in vesicle budding from the endoplasmic reticulum. Science.
1993;259(5100):1466-1468.

8. Aridor M, et al. Cargo selection by the COPII budding machinery during export from the ER.J Cell Biol. 1998;141(1):61-70.

9. Kuehn MJ, et al. COPII-cargo interactions direct protein sorting into ER-derived transport vesicles. Nature. 1998;391(6663):187-190.

10. Miller E, et al. Cargo selection into COPII vesicles is driven by the Sec24p subunit. EMBO J. 2002;21(22):6105-6113.

11. Miller EA, et al. Multiple cargo binding sites on the COPII subunit Sec24p ensure capture of diverse membrane proteins into transport vesicles. Cell. 2003;114(4):497-509.

12. Bi X, et al. Structure of the Sec23/24-Sar1 pre-budding complex of the COPII vesicle coat. Nature. 2002;419(6904):271-277.

13. Stagg SM, et al. Structural basis for cargo regulation of COPII coat assembly. Cell. 2008;134(3):474-484.

14. Miller EA, Schekman R. COPII - a flexible vesicle formation system. Curr Opin Cell Biol.
2013;25(4):420-427.

15. Paccaud JP, et al. Cloning and functional characterization of mammalian homologues of the COPII component Sec23. Mol Biol Cell. 1996;7(10):1535-1546.

16. Boyadjiev SA, et al. Cranio-lenticulo-sutural dysplasia is caused by a SEC23A mutation leading to abnormal endoplasmic-reticulum-to-Golgi trafficking. Nat Genet. 2006;38(10):1192-1197.

17. Lang MR, et al. Secretory COPII coat component Sec23a is essential for craniofacial chondrocyte maturation. Nat Genet. 2006;38(10):1198-1203.

18. Schwarz K, et al. Mutations affecting the secretory COPII coat component SEC23B cause congenital dyserythropoietic anemia type II. Nat Genet. 2009;41(8):936-940.

19. Huse M, et al. Shouts, whispers and the kiss of death: directional secretion in T cells. Nat Immunol.2008;9(10):1105-1111.

20. Jolly C, Sattentau QJ. Regulated secretion from CD4+ T cells. Trends Immunol. 2007;28(11):474-481. 
21. Fujiwara T, et al. Brefeldin A causes disassembly of the Golgi complex and accumulation of secretory proteins in the endoplasmic reticulum. J Biol Chem. 1988;263(34):18545-18552.

22. Henden AS, Hill GR. Cytokines in graft-versus-host disease. JImmunol. 2015;194(10):4604-4612.

23. Shlomchik WD. Graft-versus-host disease. Nat Rev Immunol. 2007;7(5):340-352.

24. Welniak LA, et al. Immunobiology of allogeneic hematopoietic stem cell transplantation. Annu Rev Immunol. 2007;25:139-170.

25. Khoriaty R, Vet al. The COPII pathway and hematologic disease. Blood. 2012;120(1):31-38.

26. Khoriaty R, et al. Functions of the COPII gene paralogs SEC23A and SEC23B are interchangeable in vivo. Proc Natl Acad Sci U S A. 2018;115(33):E7748-E7757.

27. Khoriaty R, et al. Pancreatic SEC23B deficiency is sufficient to explain the perinatal lethality of germline SEC23B deficiency in mice. Sci Rep. 2016;6:27802.

28. Khoriaty R, et al. Absence of a red blood cell phenotype in mice with hematopoietic deficiency of SEC23B. Mol Cell Biol. 2014;34(19):3721-3734.

29. Tao J, et al. SEC23B is required for the maintenance of murine professional secretory tissues. Proc Natl Acad Sci U S A. 2012;109(29):E2001-E2009.
30. Khoriaty R, et al. SEC23B is required for pancreatic acinar cell function in adult mice. Mol Biol Cell. 2017;28(15):2146-2154.

31. Iolascon A, et al. Congenital dyserythropoietic anemias: molecular insights and diagnostic approach. Blood. 2013;122(13):2162-2166.

32. Punzo F, et al. Congenital dyserythropoietic anemia type II: molecular analysis and expression of the SEC23B gene. Orphanet JRare Dis. 2011;6:89.

33. Heimpel H, et al. Congenital dyserythropoietic anemia type II: epidemiology, clinical appearance, and prognosis based on long-term observation. Blood. 2003;102(13):4576-4581.

34. Zhang H, et al. A case report of congenital erythropoietic anemia II in China with a novel mutation. Ann Hematol. 2019;98(4):1041-1043.

35. Mancias JD, Goldberg J. The transport signal on Sec22 for packaging into COPII-coated vesicles is a conformational epitope. Mol Cell. 2007;26(3):403-414.

36. El-Brolosy MA, Stainier DYR. Genetic compensation: a phenomenon in search of mechanisms. PLoS Genet. 2017;13(7):e1006780.

37. Liang $\mathrm{H}, \mathrm{Li}$ WH. Functional compensation by duplicated genes in mouse. Trends Genet. 2009;25(10):441-442.

38. O'Leary MN, et al. The ribosomal protein Rpl22 controls ribosome composition by directly repressing expression of its own paralog, Rpl22l1. PLoS Genet. 2013;9(8):e1003708.

39. Antonny B, Schekman R. ER export: public transportation by the COPII coach. Curr Opin Cell Biol. 2001;13(4):438-443.

40. Espenshade PJ, et al. Sterols block binding of COPII proteins to SCAP, thereby controlling SCAP sorting in ER. Proc Natl Acad Sci U S A. 2002;99(18):11694-11699.

41. Kidani Y, et al. Sterol regulatory element-binding proteins are essential for the metabolic programming of effector $\mathrm{T}$ cells and adaptive immunity. Nat Immunol. 2013;14(5):489-499.

42. Reddy P, et al. A crucial role for antigen-presenting cells and alloantigen expression in graft-versus-leukemia responses. Nat Med. 2005;11(11):1244-1249.

43. Reddy P, et al. Histone deacetylase inhibition modulates indoleamine 2,3-dioxygenase-dependent DC functions and regulates experimental graft-versus-host disease in mice. J Clin Invest. 2008;118(7):2562-2573.

44. Seki A, Rutz S. Optimized RNP transfection for highly efficient CRISPR/Cas9-mediated gene knockout in primary T cells. J Exp Med. 2018;215(3):985-997. 